\title{
Genome Comparison of Progressively Drug Resistant Plasmodium falciparum Lines Derived from Drug Sensitive Clone
}

\author{
Ravi Toteja, Lathika Nair, VK Bhasin ${ }^{+}$ \\ Department of Zoology, University of Delhi, Delhi 110007, India
}

\begin{abstract}
Chloroquine has been the mainstay of malaria chemotherapy for the past five decades, but resistance is now widespread. Pyrimethamine or proguanil form an important component of some alternate drug combinations being used for treatment of uncomplicated Plasmodium falciparum infections in areas of chloroquine resistance. Both pyrimethamine and proguanil are dihydrofolate reductase (DHFR) inhibitors, the proguanil acting primarily through its major metabolite cycloguanil. Resistance to these drugs arises due to specific point mutations in the dhfr gene. Cross resistance between cycloguanil and pyrimethamine is not absolute. It is, therefore, important to investigate mutation rates in $\mathrm{P}$. falciparum for pyrimethamine and proguanil so that DHFR inhibitor with less mutation rate is favored in drug combinations. Hence, we have compared mutation rates in $\mathrm{P}$. falciparum genome for pyrimethamine and cycloguanil. Using erythrocytic stages of $\mathrm{P}$. falciparum cultures, progressively drug resistant lines were selected in vitro and comparing their RFLP profile with a repeat sequence. Our finding suggests that pyrimethamine has higher mutation rate compared to cycloguanil. It enhances the degree of genomic polymorphism leading to diversity of natural parasite population which in turn is predisposes the parasites for faster selection of resistance to some other antimalarial drugs.
\end{abstract}

Key words: Plasmodium falciparum - malaria - drug resistance - sequencing-RFLP - dihydrofolate reductase

New malaria cases are annually increasing. This increase can be attributed partly to the development of resistance by Plasmodium falciparum to the most commonly used, inexpensive, synthetic antimalarial drug, chloroquine. Chloroquine has been the mainstay of malaria chemotherapy for the past five decades, but resistance to it is now widespread. Pyrimethamine or proguanil form an important component of some alternate drug combinations being used for treatment of uncomplicated $P$. falciparum infections in areas of chloroquine resistance. Pyrimethamine-sulfadoxine combination is usually deployed as a successor to chloroquine. Proguanil-atovaquone, a new antimalarial combination, first registered in the United Kingdom in 1996 for treatment of multidrug-resistant falciparum malaria and has subsequently been registered for this indication in 32 other countries

The work received support from the Department of Biotechnology, Government of India. RT is a recipient of Research Fellowship from Council of Scientific and Industrial Research (CSIR), India.

${ }^{+}$Corresponding author. Fax: +91-11-7257524

E-mail: virendrabhasin@hotmail.com

Received 13 November 2000

Accepted 3 January 2001 worldwide (Kremsner et al. 1999, Bustos et al. 1999). Both pyrimethamine and proguanil are dihydrofolate reductase (DHFR) inhibitors (Cowman \& Foote 1990), the proguanil acting primarily through its major metabolite cycloguanil (Armstrong \& Smith 1974). In Plasmodium DHFR is formed as DHFR-TS (thymidylate synthetase) a bifunctional protein, the enzymes acting sequentially and genes for these enzymes (dhfr-ts) are colinear (Garrett et al. 1984). Resistance to pyrimethamine and proguanil arises due to specific point mutations in the $d h f r$ gene (Peterson et al. 1988). Cross resistance between proguanil and pyrimethamine is not absolute, resistance to the two drugs being controlled by different point mutations in dhfr gene (Peterson et al. 1990, Foote et al. 1990). It is, therefore, important to investigate the rate at which $P$. falciparum parasites develop resistance to both these drugs so that DHFR inhibitor with less mutation rate is favored in a drug combination to prolong the effectiveness of such combinations. Hence, we have compared point mutations in $d h f r$ gene of progressively drug resistant $P$. falciparum lines to pyrimethamine and cycloguanil that were selected in vitro from a sensitive clone, and RFLP profile of whole genome of the selected lines with a known falciparum repeat sequence was also carried out. The other parameter studied include the predisposition, if any, for faster selec- 
tion of resistance to sulfadoxine in the parasite line highly resistant to pyrimethamine.

\section{MATERIALS AND METHODS}

Parasites - The P. falciparum isolate FCD-3 and FCD-4 obtained locally from infected patients with consent and a clone F-56 derived from FCD4 were the parasite lines used in the present study (Mehra \& Bhasin 1996). The clone was obtained by the limiting dilution method from the erythrocytic stages of the isolate cultivated continuously in vitro by the candle-jar procedure of Trager and Jensen (1976).

Stock solutions of drugs - Pyrimethamine (Sigma) $10^{-2} \mathrm{M}$ stock solution was prepared in $0.05 \%$ lactic acid and $10^{-2} \mathrm{M}$ stock of cycloguanil in $70 \%$ ethanol. Further dilutions were made in RPMI-1640 medium prior to use. Sulfadoxine $10^{-}$ ${ }^{1} \mathrm{M}$ stock solution was made in DMSO and diluted further in Waymouth MB752/1 medium, which is similar to RPMI-1640 except it does not contain para-aminobenzoic acid. Cycloguanil was a gift from ICI pharmaceuticals, UK and sulfadoxine from Lupin, India.

Susceptibility test method - Susceptibility of parasites to DHFR inhibitors and other antimalarials was determined by exposing parasites in triplicate in 24-well tissue culture plates to graded concentrations of each drug by the modified $48 \mathrm{~h}$ test method (Nguyen-Dinh \& Payne 1980). The parasite material for experiments was obtained from stock cultures and subjected to sorbitol lysis to get synchronized ring stages (Lambros \& Vanderberg 1979). The parasitemia was adjusted to less than $1 \%$ with uninfected fresh erythrocytes and this material was made to $50 \%$ cell suspension with appropriate medium. Aliquots of $20 \mu \mathrm{l}$ of the above suspension were added into series of wells of the test plate, each holding $480 \mu \mathrm{l}$ of complete medium with or without a drug, yielding final cell suspension of $2 \%$. Loaded test plates were incubated at $37^{\circ} \mathrm{C}$ in a candle-jar for $96 \mathrm{~h}$ with daily changes of medium. The drug was included in experimental wells for the first two days only. Blood smears were made at the end of 48 and $96 \mathrm{~h}$, stained with Giemsa. A minimum of 5,000 erythrocytes was enumerated to determine parasitemia in each well. Percentage reduction of parasitemia in relation to control was calculated from percentage inhibition of growth. Fifty percent, $95 \%$ and minimum complete inhibitory concentrations ( $\mathrm{IC}_{50}, \mathrm{IC}_{95}$ and MIC, respectively) were extrapolated from semilog plot of various concentrations of the drug against percent inhibition of growth obtained from averages of triplicate wells.

In vitro selection of drug resistance - The cloned line F-56 and a highly pyrimethamine re- sistant line selected in vitro were separately subjected to increasing sulfadoxine pressure in Waymouth MB752/1 medium to select a series of progressively sulfadoxine resistant lines. The parasites were subjected to their $\mathrm{IC}_{95}$ concentration of the drug for $48 \mathrm{~h}$. After $48 \mathrm{~h}$ drug was removed and the surviving parasites were allowed to multiply in drug free medium. The susceptibility of these parasites to sulfadoxine was determined. If there was no change in the sensitivity of these parasites to sulfadoxine, they were again exposed to the same $\mathrm{IC}_{95}$ concentration. The procedure was repeated till there was an increase in the $\mathrm{IC}_{50}$ to the parasites. The surviving more resistant parasites were subjected to their new $\mathrm{IC}_{95}$ concentration of sulfadoxine to select more resistant line. This protocol for selection of progressively sulfadoxine resistant parasites was repeated for each of the subsequent rounds. Selection procedure for increased DHFR inhibitor resistance has been described by Bhasin and Nair (1996).

Extraction of parasite DNA -The frozen parasite samples $\left(\sim 10^{10}\right.$ parasites $)$ were thawed, mixed with two volumes of $1 \%$ cold acetic acid and centrifuged at 6,000 rpm for $20 \mathrm{~min}$ at $4^{\circ} \mathrm{C}$. Supernatant, containing most of the haemoglobin, was removed and the pellet was resuspended in two volumes of $1 \%$ triton $\mathrm{X}-100$. It was again spun at 6,000 rpm for $20 \mathrm{~min}$ at $4^{\circ} \mathrm{C}$ and supernatant discarded. The resulting pellet was washed twice with $0.85 \%$ $\mathrm{NaCl}(\mathrm{pH} 7.4)$. The washed parasite pellet was resuspended in DNA extraction buffer $(10 \mathrm{mM}$ Tris $\mathrm{pH} 8.0 ; 0.1 \mathrm{M}$ EDTA $\mathrm{pH} 8.0 ; 20 \mu \mathrm{g} / \mathrm{ml}$ RNAase $\mathrm{A} ; 0.5 \% \mathrm{SDS}$ ) in a volume equal to that of the original frozen sample and incubated at $37^{\circ} \mathrm{C}$ for $1 \mathrm{~h}$ with the intermittent shaking. Proteinase $\mathrm{K}$ was added at concentration of $100 \mu \mathrm{g} / \mathrm{ml}$ to the above mixture, mixed gently and incubated overnight at $37^{\circ} \mathrm{C}$. DNA was extracted with an equal volume of phenol once, phenol:chloroform (1:1) twice, chloroform: isoamyl alcohol (24:1) once and chloroform twice. To the aqueous phase of the final extraction step was added 0.2 volume of $10 \mathrm{M} \mathrm{am}$ monium acetate and 2.5 vol. of cold absolute alcohol. This was left overnight at $-20^{\circ} \mathrm{C}$ for DNA to precipitate. DNA was pelleted by centrifugation at $12,000 \mathrm{rpm}$ for $30 \mathrm{~min}$ at $4^{\circ} \mathrm{C}$ and supernatant carefully removed. The pellet was washed with cold $70 \%$ ethanol and dissolved in $200 \mu \mathrm{l}$ of TE (10 $\mathrm{mM}$ Tris $\mathrm{Cl} \mathrm{pH}$ 7.5; 1 mM EDTA pH 8.0).

Polymerase chain reaction - In vitro amplification of $1.1 \mathrm{~Kb}$ domain of the dhfr-ts gene from the template, parasite DNA, was carried out by PCR (Mullis \& Faloona 1987) using the following oligonucleotide primer pairs:5-ATG ATG GAA CAA GTC TGC GAC-3/' (sense) and 5/-CAC CTA CTC CGT TCG-3/ (antisense). These synthetic 
oligonucleotides were customarily designed from the complete DNA sequence of the gene. PCR reaction mixture consisted of approximately $70 \mathrm{ng}$ of template DNA, 15 pmol of each primer, 200 $\mu \mathrm{M}$ dNTPs in a final volume of $100 \mu \mathrm{l}$ containing reaction buffer. The reaction mixture was overlaid with a drop of mineral oil, heated for $5 \mathrm{~min}$ at $94^{\circ} \mathrm{C}$ to denature the template DNA completely, while the reaction was still at $94^{\circ} \mathrm{C}, 0.5 \mu \mathrm{l}$ (5 units/ $\mu \mathrm{l})$ of Taq polymerase (Genei, India) was added. Amplification was carried out under standard conditions for 25 cycles.

Cloning PCR products and sequencing - The amplified PCR products were polished by incubating with the Klenow fragment in presence of dNTPs (Frohman 1994). The polished amplicon was purified/extracted from electrophoresed sample on $0.7 \%$ low melting agarose gel for insertion into Sma I digested pUC-19 by ligation (Sambrook et al. 1989). The ligation mix was used to transform competent JM 101 Escherichia coli cells. Recombinant white colonies were picked up from agar plates containing antibiotic, X-gal and IPTG. Plasmids from the white colony were harvested using one step plasmid isolation method and DNA analysed. Appropriate recombinant plasmid preparation was purified by $\mathrm{CsCl}$ gradient centrifugation for sequencing. DNA sequencing reactions were carried out manually by dideoxy chain termination method of Sanger et al. (1977) using Sequenase ${ }^{\mathrm{TM}}$ Version 2.0 DNA sequencing kit from $\mathrm{USB}^{\mathrm{TM}}$, as per their protocol.

RFLP by Southern hybridisation - Parasite DNA digested with Hind III, Alu I or Taq I restriction endonuclease was size fractionated on $0.8 \%$ agarose gel by electrophoresis and transferred to nylon membrane (Southern 1975). The membrane was probed with synthetic 21-oligomer, $5^{\prime}$ end labelled with ${ }^{32} \mathrm{P}$ using $\mathrm{T}_{4}$ polynucleotide kinase. The probe used for Southern hybridization had the following sequence: 5'-AGGTCTT AACTTGACTAACAT- $3^{\prime}$. This oligonucleotide is known to be repeat sequence in $P$. falciparum genome (Aslund et al. 1985). The hybridized membrane was exposed to X-ray film for autoradiogram.

\section{RESULTS}

Sensitivity of parasites to antimalarials - Susceptibility of the $P$. falciparum parasites to cycloguanil, sulfadoxine and pyrimethamine are presented in Table I. Data shows that all parasites are clinically sensitive to sulfadoxine. Clone F-56 and FCD-4 isolate are sensitive to pyrimethamine and cycloguanil as well. FCD-3 is a naturally occurring pyrimethamine resistant isolate. PR-5, a line derived from F-56 clone by in vitro selection procedure, is highly resistant to pyrimethamine.

Selection of drug resistant parasites - Progressively drug resistant falciparum lines to cycloguanil, sulfadoxine and pyrimethamine were derived from the cloned F-56 line by continuously cultivating the parasites in vitro with or without respective drug pressure for number of days (Table II). The $\mathrm{IC}_{50}$ to cycloguanil in CY-1 line was $2 \mathrm{X}$ $10^{-9} \mathrm{M}$, a decrease in sensitivity by about 75 -fold in 351 days. Only about 35 -fold decrease in sensitivity to sulfadoxine was observed at the end of 555 days of in vitro selection in SD-1 line. From susceptibility data both CY-1 and SD-1 can be regarded clinically sensitive lines to cycloguanil and sulfadoxine, respectively. Susceptibility to pyrimethamine progressively increased in various PRlines, registering an increase in 2.5 fold in 24 days (PR-1) to 2400 fold rise in $\mathrm{IC}_{50}$ value in 348 days (PR-5) of cultivation/selection, derived from F-56 sensitive clone. Susceptibility profile of PR-5 to various drugs in vitro is presented in Table I.

Selection of sulfadoxine resistance from a pyrimethamine resistant line - In pyrimethamine sensitive cloned F-56 line there was only 35 -fold decrease in sensitivity to sulfadoxine at the end of 555 days (Table II) of selection protocol. Highly pyrimethamine resistant the PR-5 line, derived from F-56, when subjected to selection pressure of sulfadoxine, the $\mathrm{IC}_{50}$ dose in vitro rose from 4 $\mu \mathrm{M}$ to $2900 \mu \mathrm{M}$, thus a 725 -fold change in susceptibility was recorded only in 363 days of selection procedure.

PCR and sequencing dhfr gene - Sufficient DNA from cryopreserved parasites of the F-56 clone, CY-1, PR-1 to PR-5 lines and FCD-3 iso-

\section{TABLE I}

In vitro susceptibility of erythrocytic stages of Plasmodium falciparum parasites to antimalarial drugs, determined by 48 -h test. The starting parasitemia was less than $1 \%$ and hematocrit $2 \%$

\begin{tabular}{lcccccc}
\hline Parasites & \multicolumn{2}{c}{ Pyrimethamine $(\mathrm{nM})$} & \multicolumn{2}{c}{ Cycloguanil $(\mathrm{nM})$} & \multicolumn{2}{c}{ Sulfadoxine $(\mu \mathrm{M})$} \\
\cline { 2 - 7 } & $\mathrm{IC}_{50}$ & $\mathrm{IC}_{90}$ & $\mathrm{IC}_{50}$ & $\mathrm{IC}_{90}$ & $\mathrm{IC}_{50}$ & $\mathrm{IC}_{90}$ \\
\hline Clone F-56 & 1.5 & 3.6 & 0.072 & 0.27 & 22 & 150 \\
Isolate FCD-4 & 1.5 & 4.6 & 0.084 & 0.68 & - & - \\
Isolate FCD-3 & 320 & 2700 & - & - & - & - \\
Line PR-5 & 3600 & 8000 & 74 & 310 & 4 & 400 \\
\hline
\end{tabular}




\section{TABLE II}

Increase in susceptibility of in vitro selected lines of Plasmodium falciparum derived from the drug sensitive parent clone, F-56 (cycloguanil $\mathrm{IC}_{50}=0.072 \mathrm{nM}$, sulfadoxine $\mathrm{IC}_{50}=22 \mu \mathrm{M}$, pyrimethamine $\mathrm{IC}_{50}=1.5 \mathrm{nM}$ ).

The parasites were under continuous cultivation for several days with or without drug pressure

\begin{tabular}{lccc}
\hline Selected line & $\begin{array}{c}\text { Days of continuous } \\
\text { cultivation/selection }\end{array}$ & $\begin{array}{c}\text { Fold increase } \\
\text { in }\left(\mathrm{IC}_{50}\right)\end{array}$ & $\begin{array}{c}\text { Clinical } \\
\text { status }\end{array}$ \\
\hline $\mathrm{CY}^{a}-1$ & 351 & 75 & CY sensitive \\
$\mathrm{SD}^{b}-1$ & 555 & 35 & SD sensitive \\
$\mathrm{PR}^{c}-1$ & 24 & 2.5 & PR sensitive \\
$\mathrm{PR}^{-2}$ & 120 & 17.3 & PR sensitive \\
$\mathrm{PR}-3$ & 204 & 140 & PR resistant \\
PR-4 & 261 & 340 & PR resistant \\
PR-5 & 348 & 2400 & PR resistant
\end{tabular}

$a$ : CY (cycloguanil); $b$ : SD (sulfadoxine); $c$ : PR (pyrimethamine)

late, was extracted. The $1.1 \mathrm{~Kb}$ domain spanning dhfr-ts genes from each of the DNA samples amplified using sense and antisense primers by PCR. The polished amplicon was cloned into pUC-19 for sequencing. The nucleotide codons obtained by sequencing at position 51, 59, 108 and 164 amino acid residues of the DHFR enzyme are presented in Table III. The nucleotide codons at the above positions were found to be similar in amplified DNA samples of F-56, CY-1, PR-1 and PR-2 lines. From in vitro susceptibility data all these were clinically pyrimethamine sensitive lines. The clinically pyrimethamine resistant parasites, the PR-3 to PR-5 in vitro selected lines and the natural isolate FCD-3, all showed point mutations leading to alterations in the codon 59 and 108 (Table III). The codon 59 had changed to CGT and 108 to AAC. In naturally occurring pyrimethamine resistance in the field isolate FCD-3, an additional mutation was found in codon 51 (AAT to ATT).

Restriction fragment length polymorphism (RFLP) analysis - DNA from the parent cloned F56 line, CY-1 and PR-1 to PR-5 lines, was each digested separately with Hind III, Alu I or Taq I restriction enzyme, size fractionated on an agarose gel and probed with a synthetic oligomer, known to be a repeat sequence in $P$. falciparum genome, by Southern hybridization. No difference was seen in the band profile of F-56, CY-1, PR-1, PR-2 or PR-3 lines (data not shown), whereas RFLP analysis clearly depicts changes in the band profile of Taq I digested DNA of PR-4 and PR-5 lines compared to F-56 (Figure). The prominent changes in band profile were detected simultaneously with the appearance of high level of pyrimethamine resistance in PR-4 and PR-5. Similar changes were also observed in an autoradiogram of Alu I digested Southern blot (data not shown).

\section{TABLE III}

Amino acid residues present in the DHFR enzyme of different parasite lines as inferred from sequencing of the dhfr gene

\begin{tabular}{lcccc}
\hline Clone/Isolate & \multicolumn{4}{c}{ Position of Amino acids } \\
\cline { 2 - 5 } & 51 & 59 & 108 & 164 \\
\hline F-56 & Asn & Cys & Ser & Ile \\
PR-1 & Asn & Cys & Ser & Ile \\
PR-2 & Asn & Cys & Ser & Ile \\
PR-3 & Asn & Arg & Asn & Ile \\
PR-4 & Asn & Arg & Asn & Ile \\
PR-5 & Asn & Arg & Asn & Ile \\
FCD-3 & Ile & Arg & Asn & Ile \\
\hline
\end{tabular}

\section{DISCUSSION}

The exposure of parasite population to submaximal inhibitory drug concentrations provides the selective pressure to resistance. The resistant mutants can survive in the presence of these intermediate levels whereas the drug sensitive parasites are killed. Repeated and intermittent exposure of the surviving parasite population to intermediate drug levels provides the optimum conditions for the emergence of resistant parasites which are biologically fit mutants to propagate. A key point mutation or multiple gene mutations may be required for a reduction of susceptibility. These mutations occur spontaneously and rarely. As they exist infrequently in parasite populations not exposed to antimalarial drugs, these mutations must be acquired and lost at a similar rate in wild parasite populations to maintain equilibrium (Mackinnon \& Hastings 1998). Thus, the chance of a resistant mutant malaria parasite being selected 


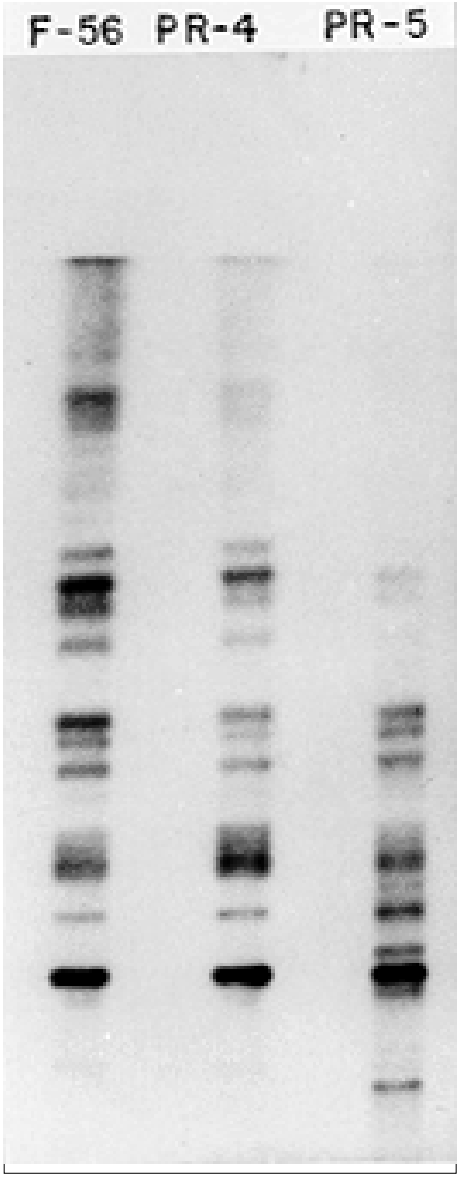

RFLP analysis of DNA digested with Taq I from F-56, PR-4 and PR-5 lines and probed with ${ }^{32} \mathrm{P}$ end labeled $5^{\circ}$ AGGTCTTAACTTGACTAACAT-3 ' primer. Prominenent is the disappearance of some bands in upper half of the autoradiogram in PR-4, PR-5 lanes and appearance of additional bands in PR-5 lane. Changed band profile is due to pyrimethamine selection

by that antimalarial drug depends on several factors, such as the total number of parasites exposed to drug pressure, the mutation frequencies, the elimination profile of the drug, and the drug susceptibility and fitness of mutants (Rosario et al. 1978). Some of these factors can not be accurately controlled by employing in vivo study systems. In vitro systems, on the other hand, provide a means to study most of these parameters under controlled conditions. By using in vitro cultivated erythrocytic stages of the cloned $P$. falciparum drug sensitive line, it was possible for us to expose the known number of genetically homogenous population of parasites to desired drug concentrations, repeatedly for fixed duration to select resistant lines and compare the rate at which parasite resistance develops to antimalarial drugs. The progressively drug resistant lines, like PR-1 to PR-5 derived from pyrimethamine sensitive cloned line F-56, form a good material to study accompanying changes at gene or genome level of the parasites.

In the present in vitro selection of drug resistant parasites, resistance to pyrimethamine developed much faster and stronger compared to cycloguanil or sulfadoxine. In 348 days continuous cultivation under pyrimethamine selection there was 2400 -fold increase in $\mathrm{IC}_{50}$ value to pyrimethamine whereas only 75 -fold decrease in sensitivity to cycloguanil was registered in 351 days cultivation under cycloguanil selection pressure (Bhasin \& Nair 1996). There was 35 -fold decrease in sensitivity to sulfadoxine in 555 days. Emergence of strains resistant to pyrimethamine appeared in the field rapidly soon after introduction of the pyrimethamine-sulfadoxine combination as an alternate to chloroquine in all countries where this combination was deployed. The resistance to pyrimethamine-sulfadoxine combination is fast increasing (Ronn et al. 1996). Proguanil, unlike pyrimethamine, still plays a useful role in prophylaxis and treatment in combination with other drugs (Peters 1998). These clinical observations are in accordance with our findings that parasites develop resistance to cycloguanil reluctantly compared to pyrimethamine.

Clinical field isolates resistant to pyrimethamine show a key point mutation leading to alteration of Ser-108 or Thr-108, found in sensitive isolates, to Asn-108 (Peterson et al. 1988) in the enzyme DHFR. Our sequencing studies also demonstrate the existence of Asn-108 point mutation in pyrimethamine resistant lines, the PR-3 to PR-5 and its absence in F-56, CY-1, PR-1 and PR2 lines, all being pyrimethamine sensitive. There was, however, no co-relation found between the number of point mutations observed in the $d h f r$ gene and decrease in pyrimethamine sensitivity in the in vitro selected lines, beyond some point. For instance, point mutations leading to Arg-59 and Asn-108 found in PR-3 remained unchanged in PR5 with no additional mutation observed in the sequenced $d h f r$ domain whereas there was substantial rise in the resistance, increasing from 140-fold to 2400 -fold. The naturally resistant FCD-3 isolate had an additional alteration isoleucine51whereas in all other lines sequenced had Asg51 residue in the DHFR. Some other factors are possibly contributing to the pyrimethamine sensitivity pattern observed. These considerations prompted us to look for alterations in the whole genome of progressively resistant falciparum lines by RFLP. 
No apparent differences were observed in band profile of F-56, CY-1, PR-1, PR-2 or PR-3 lines whereas clear and gross changes in the band profile of Taq I digested DNA of PR-4 and PR-5 lines were seen. These changes obviously occurred during selection either due to loss of existing or gain of new Taq I restriction sites, caused by pyrimethamine. The prominent changes in band profile were detected simultaneously with emergence of strong pyrimethamine resistance in the selected lines PR-4 and PR-5. The slightly resistant PR-3 line showed no change in band pattern. The RFLP also shows that pyrimethamine enhances the genomic polymorphism by random mutations in the genome of pyrimethamine resistant parasites and this may lead to even diversity of natural parasite population, which in turn might be predisposed for faster selection of resistance to other antimalarial drugs. To test this hypothesis we subjected the highly pyrimethamine resistant PR-5 line for selection to sulfadoxine resistance and compared the rate of emergence of sulfadoxine resistance with pyrimethamine sensitive F-56 line. Resistance to sulfadoxine in PR-5 line appeared much faster and stronger than F-56. In 555 days of cultivation/selection there was only 35 -fold rise in $\mathrm{IC}_{50}$ to sulfadoxine in F-56 whereas 725-fold rise was recorded in 363 days in PR-5 line. Clearly the PR-5 line was predisposed for faster selection to sulfadoxine resistance. This predisposition might be due to randomly introduced mutations by pyrimethamine in the pyrimethamine resistant line. This finding is supported by another field observation (Djimde et al. 1997) in Mali. An increase in resistant mutants from $10 \%$ to $90 \%$ of infections in 109 residents of an endemic area of Mali who received pyrimethamine alone prophylactically for five weeks. Similar, study on genes for $d h f r$ and dhps (dihydropteroate synthetase), in parasites in children in Tanzania before treatment with chloroproguanil-dapsone or pyrimethaminesulfadoxine, and in parasites recurring three weeks after treatment, showed that both drug combinations selected for mutations in $d h f r$. Pyrimethamine-sulfadoxine, but not chloroproguanildapsone, were also selected for the dhps locus (Curtis et al. 1998). In conclusion we can tentatively infer that pyrimethamine resistant falciparum parasites are selected faster than cycloguanil. The pyrimethamine resistant parasites are predisposed for faster selection of resistance to sulfadoxine. The pyrimethamine selection pressure enhances genomic diversity by introducing random mutations in the genome of pyrimethamine resistant parasites and finally proguanil is a better choice over pyrimethamine in antimalarial drug combinations.

\section{REFERENCES}

Armstrong C, Smith CC 1974. Cyclization and Ndealkylation of chloro-guanide by rabbit and rat hepatic microsomes. Toxicol Appl Pharmacol 29: 90.

Aslund L, Franzen L, Westin G, Perrson T, Wigzell H, Petterson U 1985. Highly reterated non-coding sequence in the genome of Plasmodium falciparum is composed of 21 base pair tendem repeats. J Mol Biol 185: 509-516.

Bhasin VK, Nair L 1996. In vitro selection of Plasmodium falciparum lines resistant to dihydrofolate-reductase inhibitor and cross resistance studies. Jpn J Med Sci Biol 49: 1-14.

Bustos DG, Canfield CJ, Canete-Miguel E et al. 1999. Atovaquone/proguanil compared with chloroquine and chloroquine/sulfadoxine/pyrimethamine for treatment of acute Plasmodium falciparum malaria in Philippines. J Infect Dis 179: 1587-1590.

Cowman AF, Foote SJ 1990. Chemotherapy and drug resistance in malaria. Int J Parasitol 20: 503-513.

Curtis J, Duraisingh MT, Warhurst DC 1998. In vivo selection for a specific genotype of dihydropteroate synthetase of Plasmodium falciparum by pyrimethamine-sulfadoxine but not by chloroproguanil-dapsone treatment. J Infect Dis 177: 1429.

Djimde A, Cirtese JF, Kayentao K, Diourte Y, Doumbo O, Plowe CV 1997. Rapid selection of DHFR mutations in vivo by pyrimethamine prophylaxis. $A m J$ Trop Med Hyg 57: 229.

Foote FJ, Galatis D, Cowman AF 1990. Amino acids in the dihydrofolate reductase-thymidylate synthase gene of Plasmodium falciparum involved in the cycloguanil resistance differ from those involved in pyrimethamine resistance. Proc Natl Acad Sci USA 87: 3014-3017.

Frohman MA 1994. Cloning PCR products. In KB Mullis, F Ferre, RA Gibbs (eds), Polymerase Chain Reaction, Birkhauser, Boston, p. 14-46.

Garret CE, Coderre JA, Meek TD, Garvey EP, Claman DM, Beverley SM, Santi DV 1984. A bifunctional thymidylate synthetase-dihydrofolate reductase in protozoa. Mol Biochem Parasitol 11: 257-265.

Kremsner PG, Looaresuwan S, Chulay JD 1999. Atovaquone and proguanil hydrochloride for treatment of malaria. J Travel Med (Suppl. 1): S18-S20.

Lambros C, Vanderberg JP 1979. Synchronization of Plasmodium falciparum erythrocytic stages in culture. J Parasitol 65: 418-420.

Mackinnon MJ, Hastings IM 1998. The evolution of multiple drug resistance in malaria parasites. Trans R Soc Trop Med Hyg 92: 188-192.

Mehra N, Bhasin VK 1996. In vitro gametocyte formation in Plasmodium falciparum isolates originating from a small endemic malarious area and their DNA profiling with an oligomer probe. Acta Protozool 35: 131-136.

Mullis KB, Fallona FA 1987. Specific synthesis of DNA in vitro via a polymerase chain reaction. Meth Enzymol 155: 335-350.

Nguyen-Dinh P, Payne D 1980. Pyrimethamine sensitivity in Plasmodium falciparum: determination in vitro by a modified 48-hour test. Bull WHO 58: 909- 
912.

Peters W 1998. Drug resistance in malaria parasites of animals and man. Adv Parasitol 41: 1-62.

Peterson DS, Waliker D, Wellems TE 1988. Evidence that a point mutation in dihydrofolate reductasethymidylate synthase confers resistance to pyrimethamine in falciparum malaria. Proc Natl Acad Sci USA 85: 9114-9118.

Peterson DS, Milhous WK, Wellems TE 1990. Molecular basis of differential resistance to cycloguanil and pyrimethamine in Plasmodium falciparum. Proc Natl Acad Sci USA 87: 3018-3022.

Ronn AM, Masangeni HA, Mhina J, Wernsdrofer WH, Bygbjerg IC 1996. High level of resistance of Plasmodium falciparum to sulfadoxine-pyrimethamine in children on Tanzania. Trans R Soc Trop Med Hyg
90: 179.

Rosario VE, Hall R, Walliker D, Beale GH 1978. Persistence of drug-resistant malaria parasites. Lancet 1: 185-187.

Sambrook J, Fritch EF, Maniatis T 1989. Molecular Cloning: A Laboratory Manual, 2nd ed., Cold Spring Harbor Laboratory Press, USA.

Sanger FS, Nicklen S, Coulson AR 1977. DNA sequencing with chain terminating inhibitors. Proc Natl Acad 74: 5463.

Southern EM 1975. Detection of specific sequences among DNA fragments separated by gel electrophoresis. J Mol Biol 98: 503.

Trager W, Jensen JB 1976. Human malaria parasites in continuous culture. Science 193: 673-675. 